\title{
Ultra-compact terahertz plasmonic wavelength diplexer
}

\author{
Mingrui Yuan, ${ }^{1}$ Qingwei Wang, ${ }^{1,2}$ Yanfeng LI, $^{1, *}$ XiXIAng Zhang, ${ }^{2}$ Jiaguang \\ HAN, ${ }^{1, *}$ AND WEILI ZHANG ${ }^{3}$ \\ ${ }^{1}$ Center for Terahertz Waves and College of Precision Instrument and Optoelectronics Engineering, Key Laboratory of Optoelectronics \\ Information and Technology (Ministry of Education of China), Tianjin University, Tianjin 300072, China \\ 2 Physical Science and Engineering Division, King Abdullah University of Science and Technology, Thuwal 23955-6900, Saudi Arabia \\ ${ }^{3}$ School of Electrical and Computer Engineering, Oklahoma State University, Stillwater, Oklahoma 74078, USA \\ *Corresponding author: yanfengli@tju.edu.cn; jiaghan@tju.edu.cn
}

Received XX Month XXXX; revised XX Month, XXXX; accepted XX Month XXXX; posted XX Month XXXX (Doc. ID XXXXX); published XX Month XXXX

Terahertz (THz) spoof surface plasmon polariton (SPP) waveguides can provide subwavelength confinement, which makes it possible for the THz waves to transmit at low loss over long distances along a metallic surface. In this work, an ultra-compact wavelength diplexer formed by THz spoof SPP waveguiding structures is reported on the design and actualization. By adding a certain number of periodic pillars in the coupling part of the directional coupler, the refractive index of the anti-symmetrically distributed odd modes can be engineered, thereby adjusting the coupling length. By adjusting the periodic pillar parameters properly, the SPP modes at two target frequencies will be coupled in the device for an odd or even number of times, so that SPP modes at these two frequencies can be coupled out from different ports. The length of the wavelength diplexer is $1.6 \mathrm{~mm}$, which is about $12.8 \%$ of its traditional counterpart. Minimum simulated transmittances of $-24.34 \mathrm{~dB}$ and $-26.27 \mathrm{~dB}$ can be obtained at 0.637 THz and 0.667 THz, respectively. The insertion losses at the two operating frequencies are less than $0.46 \mathrm{~dB}$, and the extinction ratios are both better than $19 \mathrm{~dB}$. By cascading the proposed diplexers, a compact wavelength demultiplexer with more channels can be obtained, which has important applications for future THz integrated communication systems. (C) 2020 Optical Society of America

\section{INTRODUCTION}

Terahertz (THz) technology has great potential in the development of next-generation and ultra-high-speed communications due to its wide frequency band and ability to carry extremely large amounts of information $[1,2]$. The development of THz on-chip system based on surface plasmons is considered an important way for $\mathrm{THz}$ systems to become compact and multifunctional [3]. Surface plasmon polaritons (SPPs) [4-6] are localized surface waves in the optical frequency range, which are caused by collective oscillations of electrons coupled with the electromagnetic fields at a metal/dielectric interface. With their notable ability of subwavelength confinement and strong field enhancement, SPPs have found a variety of applications in the fields of superresolution imaging [7], high-density optical data storage [8], and sensitive biosensing [9-12]. In order to achieve subwavelength waveguides and compact integrated circuits, the excitation and control of SPPs are important for both optical frequency and lower frequencies. However, at the technologically important $\mathrm{THz}$ frequencies, most metals have perfect electrical conductivity and therefore their surfaces do not support well-confined modes of THz surface waves [13]. In an effort to realize highly-confined SPPs at microwave and THz frequencies, corrugated metal structures are proposed to support and propagate SPPs, known as the concept of spoof SPPs [14-16]. Many complex structures that support highlyconfined spoof SPPs have been proposed, such as corrugated metal wires [17], dominoes [18], grooved or wedged wires [19]. Among them, the domino-like metallic structure is comprised of a series of pillars arranged on the metal surface, and these units support the propagation of localized surface waves [20]. Following this structure, many studies focus on devices based on $\mathrm{THz}$ spoof SPPs have been proposed, including waveguides [21-24], directional couplers [25], low-loss crossings [26], and cured waveguiding devices [27]. Based on the above research, $\mathrm{THz}$ on-chip systems based on surface plasmons have become a promising platform to help the development and application of wireless communications in the future.

Wavelength division multiplexing (WDM) is an effective technique to achieve a rapidly growing demand for largecapacity data transmission. Wavelength division multiplexers play a significant role in the optical transmission systems of the WDM technology and are an important part of the future $\mathrm{THz}$ 
integrated circuits. In particular, in the wide fiber-to-the-X applications, demultiplexers operating at two frequencies, namely wavelength diplexers, have received special attention. At present, several schemes have been proposed for multiplexing signals at two different wavelengths, such as diffraction grating couplers coupling two wavelengths to different directions [28, 29], on-chip microring resonators for filtering different wavelengths [30], and duplexers based on multimode interference $[31,32]$. For optical devices, compactness and high integration are among the key points to be considered. In order to shorten the device length, particle swarm optimization and reverse design algorithm are also proposed [33, 34]. In addition, directional couplers [35] have been proved to be useful for filtering and separation of frequencies, which is essential in the implementation of demultiplexing components [36, 37]. The development of $\mathrm{THz}$ on-chip systems based on surface plasmons is considered the most promising solution to realize the simultaneous transmission of electrical and optical signals, which will help the $\mathrm{THz}$ systems play an important role in future communications. Meanwhile, wavelength diplexers have great potential applications in the field of ultra-high-speed information processing. However, designing a low-loss and broadband integrated passive diplexer with a wellknit structure and low manufacturing tolerances is still complicated. Moreover, as far as we know, there has been no report on the wavelength demultiplexer based on $\mathrm{THz}$ spoof SPP waveguides. Integrable wavelength diplexers with high performance that can combine/split two frequencies are a very important component for $\mathrm{THz}$ on-chip systems.

In this work, we design and achieve a wavelength diplexer based on THz spoof SPP waveguides with an ultra-compact structure. The basic principle of the device is to control the coupling lengths at two wavelengths by adding a periodic column structure in the coupling region. By properly adjusting the parameters of the periodic columns, two different frequencies can be obtained for a fixed length of the device. The length of the wavelength diplexer is $1.6 \mathrm{~mm}$, which is about $12.8 \%$ of its traditional counterpart [25]. Minimum transmittances of $-24.34 \mathrm{~dB}$ and $-26.27 \mathrm{~dB}$ can be obtained at $0.637 \mathrm{THz}$ and $0.667 \mathrm{THz}$, respectively. The insertion losses at the two operating frequencies are less than $0.46 \mathrm{~dB}$, and the extinction ratios are both better than $19 \mathrm{~dB}$. The wavelength diplexer is demonstrated to accomplish multiplexing of two different wavelengths successfully by using a fiber-optic scanning near-field THz microscopy system [26, 27]. The parameter variation, production process and operating principle of the diplexer are presented and then verified by experiments. This structure with advantages of small size and low loss is helpful for large-scale interconnection of $\mathrm{THz}$ onchip systems and minimization of the device space of complex planar $\mathrm{THz}$ integrated systems in the future.

\section{RESULTS AND ANALYSIS}

The schematic diagram of the proposed diplexer is shown in Fig. 1. This diplexer consists of spoof SPP waveguides based on the domino structure $[18,25]$, which is composed of a periodic arrangement of metallic pillars with width $w=120 \mu \mathrm{m}$, length $l=50 \mu \mathrm{m}$, and height $h=80 \mu \mathrm{m}$ separated on the top of a metallic surface with a period $p=100 \mu \mathrm{m}$. The device consists of an input waveguide, an interaction region, and two output waveguides. The interaction region includes two parallel straight waveguides, the length of the parallel part is $L$, and the gap between them is $g$. In the middle of the interaction region, a grating with a certain number of periodic pillars is implemented. These pillars are connected to the two waveguides and have the same height and period as those in the waveguides. Here, we choose the interaction region width $g$ $=120 \mu \mathrm{m}$ and the pillar width $a=20 \mu \mathrm{m}$. S-bend waveguides based on the cosine function are attached to the output port for decoupling [38]. It is worth noting that the two waves with wavelengths $\lambda_{1}$ and $\lambda_{2}$ are coupled into the upper port in Fig. $1(\mathrm{a})$, where the lower port has been made shorter for easy discrimination. For practical applications, these two ports can be made the same and the two waves can be coupled into any of those two input ports, and the device performance is guaranteed by the symmetry of the structure. The whole design with detailed structural parameters is provided in Fig. 1(b), where we define the output port on the same waveguide with the input port as the bar port, and the mode output from the bar port after coupling for an even number of times is the bar state. On the contrary, the output port of the other waveguide is called the cross port, and the mode output from the cross port after coupling for an odd number of times is the cross state.
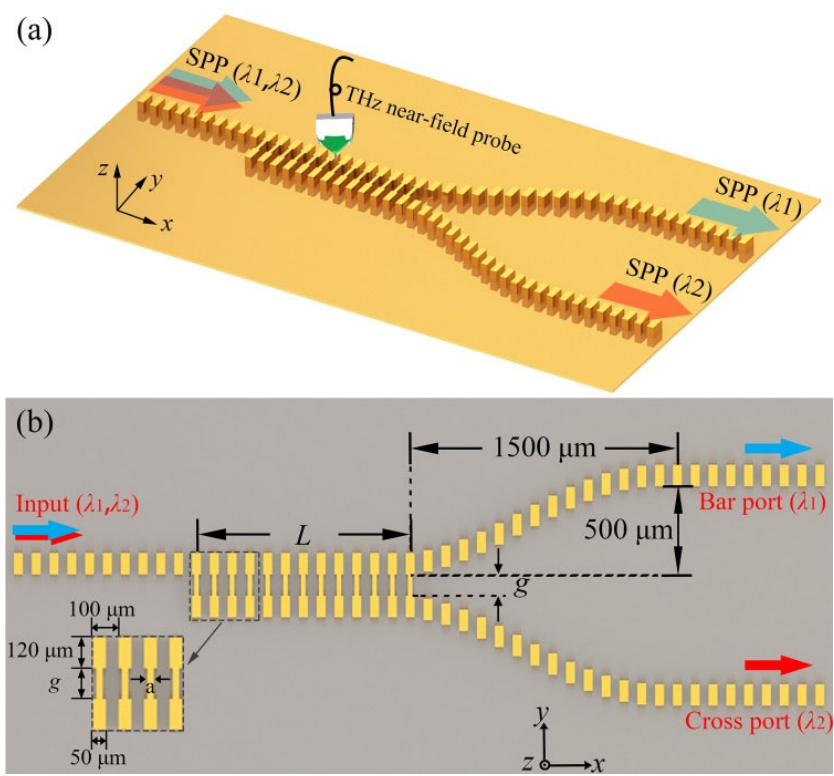

Fig. 1. (a) Three-dimensional view and (b) Two-dimensional detailed view of the wavelength diplexer. The arrows in different colors depict the propagation direction of the SPPs with two different wavelengths.

The conventional directional coupler realizes the directional transfer of mode power through the accumulation of the phase difference. This method has been used to design and implement an effective directional coupler at an operating frequency of $0.6 \mathrm{THz}$ [25]. When two parallel waveguides are close to each other, the composed structure will support two modes. Because of the presence of the grating, the even and odd modes in the interaction region will be modified, resulting in different coupling lengths at different frequencies. By properly designing the length of the coupling region, surface waves at different frequencies can be obtained from different output ports. In order to split the waves at two frequencies $f_{1}$ and $f_{2}$, the length $L$ of the interaction section needs to satisfy the following equation: 


$$
L=m L\left(f_{1}\right)=(m+n) L\left(f_{2}\right),
$$

where $m$ is a positive integer and $n$ is an odd integer that is usually set to 1 . The coupling length at frequency $f$ can be expressed as:

$$
L(f)=\pi /\left(k_{\mathrm{e}}-k_{\mathrm{o}}\right),
$$

which is the length required to shift the mode power completely from one waveguide to the other. Here, $k_{\mathrm{e}}$ and $k_{\mathrm{o}}$ are the propagation constants of the even and odd modes supported by two parallel waveguides, respectively.

In Fig. 2(a), for the conventional directional coupler, the normalized electric distributions $\left(E_{z}\right)$ of the two modes for the $y z$ (upper) and $x y$ (lower) cross-sections are displayed. To obtain the dispersion relations of the two eigenmodes, the eigenmode solver of the commercial software CST Microwave Studio is adopted. The corresponding normalized electric distributions $\left(E_{z}\right)$ for the two modes propagating in the interaction region with periodic pillars are shown in Fig. 2(b). The field distribution of the even mode is similar to that of two adjacent waveguides. But some qualitative differences in the odd mode determine the difference between the two structures. The inserted periodic pillars in the middle affect mainly the equivalent refractive index of the anti-symmetrically distributed odd mode, since it has a change in the distribution of intensity, that is, the intensity in the middle is zero, while the even mode is less influenced. Therefore, the coupling length can be adjusted according to Equation 2.

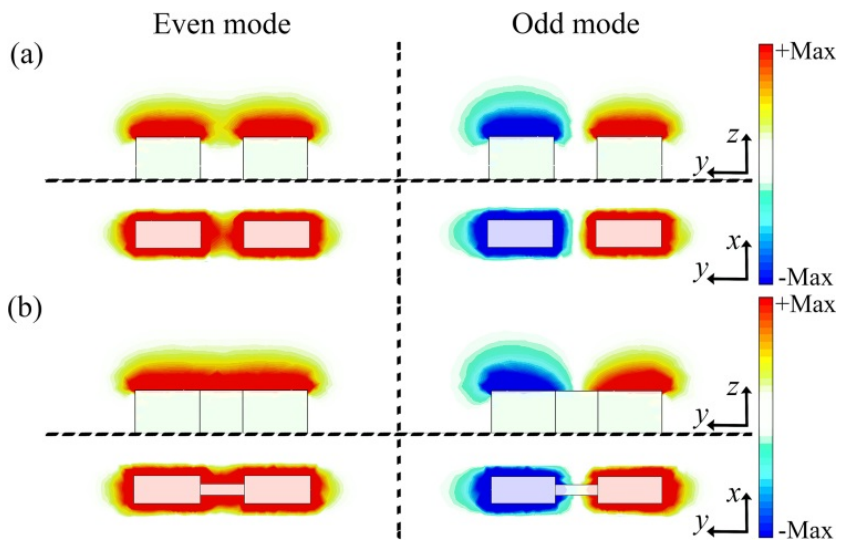

Fig. 2. Normalized electric component $\left(E_{z}\right)$ distributions for the $y z$ (upper) and $x y$ (lower) cross-sections of even (left) and odd (right) modes supported by (a) two adjacent waveguides and (b) two parallel waveguides with inserted grating.

Figure 3(a) shows the propagation constants of the even and odd modes as a function of frequency for the two cases. In the numerical simulations, the periodic boundary conditions are used to simulate a unit cell of the periodic structure. The bottom and pillars of the waveguide are assumed as perfect electrical conductors, and this setting is suitable for metals in the microwave and $\mathrm{THz}$ regions. The phase $\theta$ along the propagation direction is calculated from $0^{\circ}$ to $180^{\circ}$, and the values of the propagation constant $k_{x}$ can be calculated as $k_{x}=$ $\theta \times \pi /(180 \times p)[18]$. In the figure, the black lines indicate the dispersion relations of the two supermodes for conventional parallel waveguides, while the red lines are for the parallel waveguides with the grating. As can be seen, for the even mode, the existence of grating has little effect on the dispersion relation. However, for the odd mode, $k_{0}$ becomes smaller at the same frequency. Using Equation 2, the coupling length as a function of frequency from 0.5 to $0.65 \mathrm{THz}$ can be obtained as in Fig. 3(b). It is observed that for parallel waveguides, the coupling length has a weak dependence on frequency from 0.55 to $0.65 \mathrm{THz}$. However, the presence of the grating makes the coupling length $L(f)$ strongly frequency-dependent. Consequently, a cross-state (odd multiple of $L(f)$ ) for $f_{1}$ and a bar-state (even multiple of $L(f)$ ) for $f_{2}$ can be acquired in a short distance by carefully tailing the length of the interaction section.

Figures 3(c) and 3(d) shows the results of the coupling length as functions of $a$ and $g$, respectively. The geometrical parameters $a$ and $g$ are two independent parameters that can affect the dispersion relation of the odd mode for the waveguide but only slightly. However, in the frequency range from 0.5 to $0.65 \mathrm{THz}$, the changes of $a$ and $g$ have little effect on the dispersion curve. Therefore, the plots of the coupling length as functions of $a$ and $g$ do not show much variation. Consequently, the diplexer has strong robustness to the fabrication errors of the grating size. The values of $a$ and $g$ are first varied in the simulations and then selected such that a combination of the two parameters would lead to a minimum coupling length at the target frequency and the relationship in Eq. 1 is satisfied at the same time. Here, the optimal values of $a$ and $g$ are found to be $20 \mu \mathrm{m}$ and $120 \mu \mathrm{m}$, where the respective coupling lengths at $0.637 \mathrm{THz}$ and $0.667 \mathrm{THz}$ are $\sim 0.53 \mathrm{~mm}$ and $\sim 0.40 \mathrm{~mm}$, resulting in $m=3$. Briefly speaking, $m=3$ and $n=1$ in Equation 1 are chosen to make the following relation satisfied: $1.6 \mathrm{~mm} \approx$ $3 L$ at $0.637 \mathrm{THz} \approx 4 L 0.667 \mathrm{THz}$ ). Hence, waves at $0.637 \mathrm{THz}$ and $0.667 \mathrm{THz}$ will be obtained at the cross port and bar port, respectively.
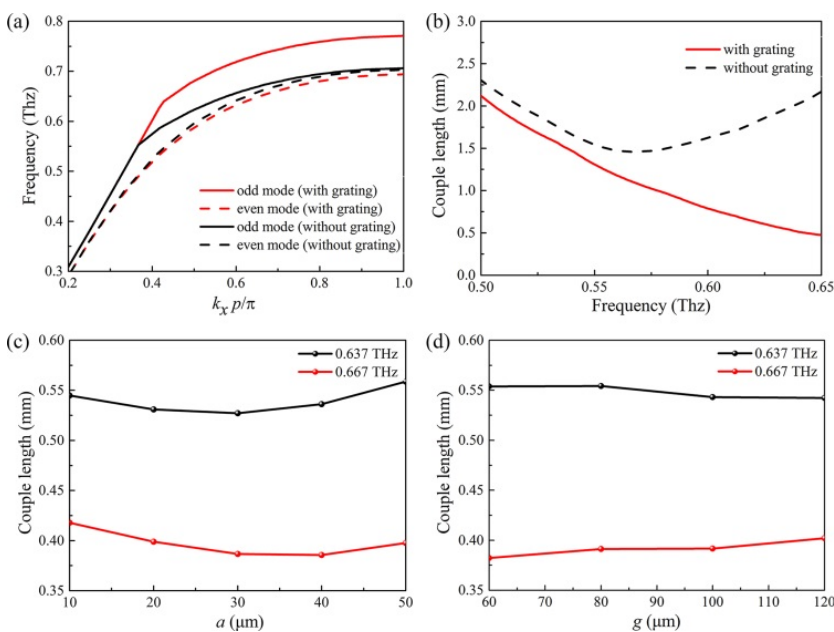

Fig. 3. (a) Dispersion relation of even and odd modes for two parallel waveguides and the same structure with inserted grating. (b) Calculated coupling length for the two structures in (a). Calculated coupling length $L(f)$ for the proposed wavelength diplexer as functions of (c) grating width $a$ and (d) gap width $g$.

To verify the functions of the wavelength diplexer, simulations based on the time domain solver of CST Microwave Studio are performed. As the source, the SPPs are excited by a waveguide port. Figures 4(a) and 4(b) show the simulated results for the normalized power $\left|E_{z}\right|^{2}$ distributions of the diplexer with a scanning area of $5 \mathrm{~mm} \times 3 \mathrm{~mm}$ for (a) $f=0.637$ $\mathrm{THz}$ and (b) $f=0.667 \mathrm{THz}$. It can be clearly observed that the mode interference occurs in the interaction region. It is also 
noted that the cross-state at $0.637 \mathrm{THz}$ and the bar-state at $0.667 \mathrm{THz}$ are realized at the end of the interaction region and subsequently waves at these two frequencies propagate out from the cross and bar ports, confirming the wavelength demultiplexing function of the device. The interaction region is three times of the coupling length at $0.637 \mathrm{THz}$ and four times of the coupling length at $0.667 \mathrm{THz}$.
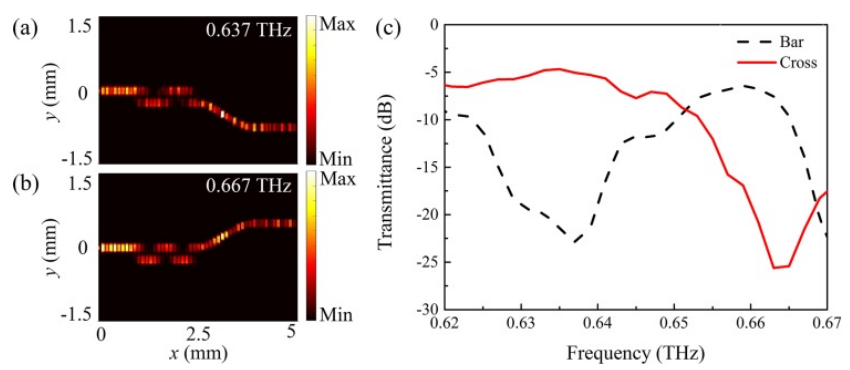

Fig. 4. Simulated results for normalized power $\left|E_{z}\right|^{2}$ distributions corresponding to (a) $f=0.637 \mathrm{THz}$ and (b) $f=0.667 \mathrm{THz}$ inputs in a horizontal plane slightly above (at $100 \mu \mathrm{m}$ ) the surface of each structure. (c) Transmittance spectra as a function of frequency for bar port (black dashed line) and cross port (red solid line), respectively.

As a comparison, a conventional directional coupler with the same parameters but lacking the grating structure is also calculated and analyzed. The coupling lengths at $0.637 \mathrm{THz}$ and $0.667 \mathrm{THz}$ are $\sim 2.08 \mathrm{~mm}$ and $\sim 2.46 \mathrm{~mm}$, respectively, leading to an interaction region as long as $12.48 \mathrm{~mm}$ to distinguish these two waves ( $m=6$ and $n=-1$ ). This means that the length of the proposed wavelength diplexer is only about $12.8 \%$ of its traditional counterpart.

The transmittance spectra are shown in Fig. 4(c). Minimum transmittances of $-24.34 \mathrm{~dB}$ and $-26.27 \mathrm{~dB}$ can be acquired at $0.637 \mathrm{THz}$ and $0.667 \mathrm{THz}$, respectively. The performance of the proposed wavelength diplexer is assessed by the extinction ratio $(E R)$ and insertion loss $(I L)$. The $E R$ is extracted as the ratio of the power output from the cross (bar) port to the bar (cross) port at $0.637 \mathrm{THz}(0.667 \mathrm{THz}): E R=10 \log \left(P_{1} / P_{2}\right)$, whereas the $I L$ is calculated by comparing the total loss of the structure with that of a straight waveguide of the same length. In other words, the difference between the total loss of the structure and that of the straight waveguide is attributed to the $I L$ of the wavelength diplexer. The loss is calculated as the ratio of the input power to the output power of the fundamental mode coupled to the waveguide: $\operatorname{Loss}=10 \log \left(P_{\text {in }} / P_{i}\right), i=1,2$. Values of $I L$ of $0.38 \mathrm{~dB}$ and $0.46 \mathrm{~dB}$ can be acquired at 0.637 $\mathrm{THz}$ and $0.667 \mathrm{THz}$, respectively, and the $E R$ values for the two frequencies are $19.18 \mathrm{~dB}$ and $19.06 \mathrm{~dB}$, respectively. The power is calculated by integrating the longitudinal component of the Poynting vector in perpendicular planes near the input of the waveguide and the output sides of the diplexer. The integrating regions have the same size of $400 \mu \mathrm{m} \times 400 \mu \mathrm{m}$, which is selected to be greater than the maximum size of the mode to ensure the correct calculation of the power distribution. In addition, since the coupling length decreases monotonically with increasing frequency as seen from Fig. 3(b), by properly designing the length of the coupling region, the proposed device can work in the whole SPP transmission range. With a larger value of $m$ in Equation 1, two closer frequencies can be obtained. The proposed wavelength diplexer can be fabricated simultaneously with the waveguides through one etching step without additional materials and processing [25]. Firstly, the waveguide structure of the diplexer is obtained by optical lithography and deep reactive ion etching on a 2-mm-thick silicon wafer. Then, the whole silicon structure is metallized in a gold sputter coater. The thickness of gold is $200 \mathrm{~nm}$, which is selected to be larger than the penetration depth of the $\mathrm{THz}$ waves in the metal. Figure 5 shows the scanning electron microscopy (SEM) image of the fabricated wavelength diplexer. The free-space THz radiation is coupled to the SPP wave by momentum matching through an arc-shaped hole array. The curved holes with a width of $40 \mu \mathrm{m}$ are arranged along the radial direction with a period of $400 \mu \mathrm{m}$. The innermost and outermost radii of the annular sectors are 2220 and $3820 \mu \mathrm{m}$ respectively, and the central angle is $60^{\circ}$. To fully utilize the excited SPPs, a fan-shaped metasurface composed of the same metallic columns as in the waveguides are designed to guide the excited SPPs to the waveguide [26, 27]. The lower-left inset of Fig. 5 shows the SEM image of the fabricated arc-shaped curved hole array and the fan-shaped metasurface. The lowermiddle inset of Fig. 5 shows the grating structure in the coupling region, and the lower-right inset shows the fabricated domino waveguide.

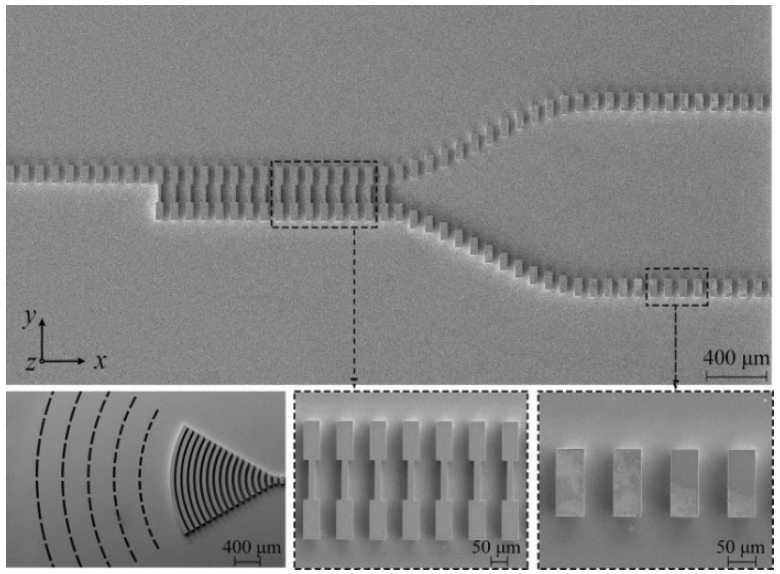

Fig. 5. SEM images of the fabricated wavelength diplexer. The lower left inset shows the SEM image of the arc-shaped curved hole array excitation region and the fan-shaped metasurface. The lower middle inset shows the grating structure in the coupling region. The lower right inset shows the domino waveguide.

The electric near-field of the SPPs is measured by fibercoupled scanning nearfield $\mathrm{THz}$ microscopy system [25-27]. With this system, THz waves incident from the bottom of the sample propagate along the $z$-direction to the excitation grating area and then excites the SPPs. The direction of the linearlypolarized $\mathrm{THz}$ wave is perpendicular to the hole gratings and parallel to the propagation axis of the waveguide so as to meet the excitation conditions of the SPPs. The excited SPPs are then detected by a near-field probe with a resolution of $8 \mu \mathrm{m}$. The $\mathrm{THz}$ probe is placed at a distance of $100 \mu \mathrm{m}$ above the sample, and a two-dimensional translation detector is used to move the probe along the $x$ - and $y$-directions. The probe scans the signals point by point with a step of $150 \mu \mathrm{m}$ along the $x$-direction and $200 \mu \mathrm{m}$ along the $y$-direction.

For the visualization of the SPP near-field, the normalized power $\left|E_{z}\right|^{2}$ distributions at the considered frequency are plotted as a two-dimension color map. Figure 6(a) shows the measured SPP field distributions on the proposed wavelength diplexer at $0.63 \mathrm{THz}$ and $0.66 \mathrm{THz}$, respectively. A strong 
electric field in the area around $y=-750 \mu \mathrm{m}$ is observed at 0.63 $\mathrm{THz}$, corresponding to the cross port of the device. Meanwhile, there is almost no energy distribution around the bar port. Again, a strong electric field in the area around $y=600 \mu \mathrm{m}$ can be obtained at $0.66 \mathrm{THz}$, which corresponds to the bar port of the device. The experimental results obtained agree well with the simulations shown in Figs. 4(a) and 4(b), which indicate that the wavelength diplexer can accomplish successful wave multiplexing. In Fig. 6(b), the normalized cross-sectional power distributions in the experiment are displayed at the ends of the S-bend waveguides as illustrated by the dotted lines (at $x=5.5$ $\mathrm{mm}$ ) in Fig. 6 (a). For the recorded power distribution, the fields are strongly concentrated in the cross port near $y=-750$ $\mu \mathrm{m}$ at $0.63 \mathrm{THz}$ and the bar port near $y=600 \mu \mathrm{m}$ at $0.66 \mathrm{THz}$. The amplitude of the field decreases rapidly when it is far away from the waveguide. Therefore, the multiplexing performance of this component is thus fully verified. Compared with the simulation results, the deterioration of the measured $E R$ values can be attributed to the resolution of the experimental measurement system: The probe collects signals point by point on the sample surface, and the collected time-domain signals can depict the propagation process of the SPPs at different frequencies by Fourier transform. The required operation time for the wavelength diplexer is about $75 \mathrm{ps}$, and the frequency resolution after Fourier transform is $15 \mathrm{GHz}$, which makes it difficult to distinguish the signal on the $\mathrm{GHz}$ order. Although zero padding can be used to increase the data length, this method does not increase the effective information of the timedomain signal, so it does not change the resolution of the fast Fourier transform. Notwithstanding the limit in measurement, the structure still performs remarkably well near the designed frequencies.
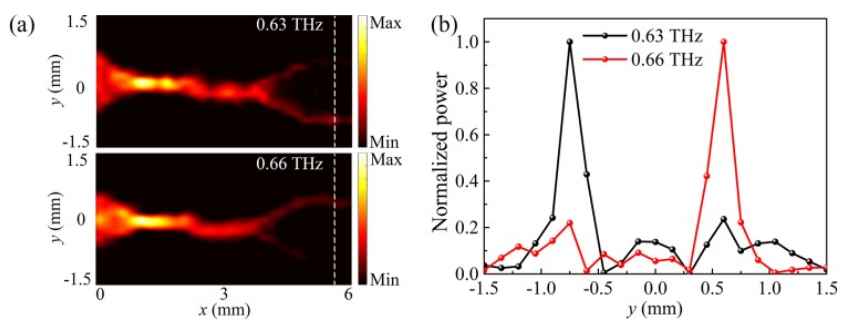

Fig. 6. Performance of the wavelength diplexer. (a) Experimental results for normalized power $\left|E_{z}\right|^{2}$ distributions corresponding to $f=$ $0.63 \mathrm{THz}$ and $f=0.66 \mathrm{THz}$ inputs in a horizontal plane slightly above (at $100 \mu \mathrm{m})$ the surface of the structure. (b) Normalized power distributions of the experimental cross-section at the output ports (line $x=5.5 \mathrm{~mm}$ ) in (a).

\section{CONCLUSION}

In conclusion, a plasmonic wavelength diplexer with ultracompact size, low loss, and high extinction ratio is proposed. A grating region is designed to control the coupling length at the two operating frequencies and is as short as $1.6 \mathrm{~mm}$, which is only $\sim 12.8 \%$ that of a conventional directional coupler. The insertion losses at the two operating frequencies are both less than $0.46 \mathrm{~dB}$ and the extinction ratios are better than $19 \mathrm{~dB}$. By properly designing the length of the coupling region, the proposed device can work in the whole SPP transmission range. The diplexer is sensitive to the length of the coupling region but has a low requirement for the size of the waveguide, so it has strong robustness to the fabrication errors of the waveguide and grating. In addition, by cascading the proposed diplexers, it is possible to develop compact wavelength demultiplexers that can handle more channels. These components will be of great value for future $\mathrm{THz}$ communication applications.

Funding. This work was funded by National Key Research and Development Program of China (2017YFA0701004); National Natural Science Foundation of China (61935015, 61875150, 61605143, 61735012, 61722509, and 61871212); Tianjin Municipal Fund for Distinguished Young Scholars (18JCJQJC45600); and King Abdullah University of Science and Technology (KAUST) Office of Sponsored Research (OSR) (OSR2016-CRG5-2950).

Disclosures. The authors declare no conflicts of interest.

\section{References}

1. S. Ummethala, T. Harter, K. Koehnle, Z. Li, S. Muehlbrandt, Y. Kutuvantavida, J. Kemal, P. Marin-Palomo, J. Schaefer, A. Tessmann, S. Garlapati, A. Bacher, L. Hahn, M. Walther, T. Zwick, S. Randel, W. Freude, and C. Koos, "THz-to-optical conversion in wireless communications using an ultra-broadband plasmonic modulator", Nat. Photonics 13, 519-524 (2019).

2. T. Nagatsuma, G. Ducournau, and C. Renaud, "Advances in terahertz communications accelerated by photonics", Nat. Photonics 10, 371-379 (2016).

3. X. Zhang, Q. Xu, L. Xia, Y. Li, J. Gu, Z. Tian, C. Ouyang, J. Han, and W. Zhang, "Terahertz surface plasmonic waves: a review", Advanced Photonics 2, 014001 (2020).

4. T. Ebbesen, H. Lezec, H. Ghaemi, T. Thio, and P. Wolff, "Extraordinary optical transmission through sub-wavelength hole arrays", Nature 391, 667-669 (1998).

5. W. Barnes, A. Dereux, and T. Ebbesen, "Surface plasmon subwavelength optics", Nature 424, 824-830 (2003).

6. S. Bozhevolnyi, V. Volkov, E. Devaux, and T. Ebbesen, "Channel plasmonpolariton guiding by subwavelength metal grooves", Phys. Rev. Lett. 95, 046802 (2005).

7. X. Zhang and Z. Liu, "Superlenses to overcome the diffraction limit", Nature Materials 7, 435-441 (2008).

8. C. Genet and T. Ebbesen, "Light in tiny holes", Nature 445, 39-46 (2007).

9. S. Nie, "Probing single molecules and single nanoparticles by surfaceenhanced raman scattering", Science 275, 1102-1106 (1997).

10. H. Yoshida, Y. Ogawa, Y. Kawai, S. Hayashi, A. Hayashi, C. Otani, E. Kato, F. Miyamaru, and K. Kawase, "Terahertz sensing method for protein detection using a thin metallic mesh", Appl. Phys. Lett. 91, 253901 (2007).

11. W. Barnes, A. Dereux, and T. Ebbesen, "Surface plasmon subwavelength optics", Nature 424, 824-830 (2003).

12. C. Huang and Y. Zhu, "Plasmonics: manipulating light at the subwavelength scale", Active and Passive Electronic Components 2007, 30946 (2007).

13. T. Jeon and D. Grischkowsky, "THz Zenneck surface wave ( $\mathrm{THz}$ surface plasmon) propagation on a metal sheet", Appl. Phys. Lett. 88, 061113 (2006).

14. F. Garcia-Vidal, L. Martín-Moreno, and J. Pendry, "Surfaces with holes in them: new plasmonic metamaterials", J. Opt. A: Pure Appl. Opt. 7, S97S101 (2005)

15. J. Pendry, "Mimicking surface plasmons with structured surfaces", Science 305, 847-848 (2004).

16. L. Liu, Z. Li, C. Gu, B. Xu, P. Ning, C. Chen, J. Yan, Z. Niu, and Y. Zhao, "Smooth bridge between guided waves and spoof surface plasmon polaritons", Opt. Lett. 40, 1810-1813 (2015) 
17. S. Maier, S. Andrews, L. Martín-Moreno, and F. García-Vidal, "Terahertz surface plasmon-polariton propagation and focusing on periodically corrugated metal wires", Phys. Rev. Lett. 97, 176805 (2006).

18. D. Martin-Cano, M. Nesterov, A. Fernandez-Dominguez, F. Garcia-Vidal, L. Martin-Moreno, and E. Moreno, "Domino plasmons for subwavelength terahertz circuitry", Opt. Express 18, 754-764 (2010).

19. A. Fernández-Domínguez, C. Williams, F. García-Vidal, L. Martín-Moreno, S. Andrews, and S. Maier, "Terahertz surface plasmon polaritons on a helically grooved wire", Appl. Phys. Lett. 93, 141109 (2008).

20. E. Brock, E. Hendry, and A. Hibbins, "Subwavelength lateral confinement of microwave surface waves", Appl. Phys. Lett. 99, 051108 (2011).

21. Z. Li, L. Liu, H. Sun, Y. Sun, C. Gu, X. Chen, Y. Liu, and Y. Luo, "Effective surface plasmon polaritons induced by modal dispersion in a waveguide", Phys. Rev. Appl. 7, 044028 (2017).

22. A. Fernández-Domínguez, E. Moreno, L. Martín-Moreno, and F. GarcíaVidal, "Guiding terahertz waves along subwavelength channels", Phys. Rev. B 79, 233104 (2009).

23. A. Fernández-Domínguez, E. Moreno, L. Martín-Moreno, and F. GarcíaVidal, "Terahertz wedge plasmon polaritons", Opt. Lett. 34, 2063 (2009).

24. G. Kumar, S. Li, M. Jadidi, and T. Murphy, "Terahertz surface plasmon waveguide based on a one-dimensional array of silicon pillars", New J. Phys. 15, 085031 (2013).

25. Y. Zhang, Y. Xu, C. Tian, Q. Xu, X. Zhang, Y. Li, X. Zhang, J. Han, and W. Zhang, "Terahertz spoof surface-plasmon-polariton subwavelength waveguide", Photonics Res. 6, 18-23 (2017).

26. M. Yuan, Y. Li, Y. Lu, Y. Zhang, Z. Zhang, X. Zhang, X. Zhang, J. Han, and W. Zhang, "High-performance and compact broadband terahertz plasmonic waveguide intersection", Nanophotonics 8, 1811-1819 (2019).

27. M. Yuan, Y. Lu, Y. Zhang, Z. Zhang, Y. Li, H. Liu, X. Zhang, J. Han, and W. Zhang, "Curved terahertz surface plasmonic waveguide devices", Opt. Express 28, 1987-1998 (2020).

28. G. Roelkens, D. Van Thourhout, and R. Baets, "Silicon-on-insulator ultracompact duplexer based on a diffractive grating structure", Opt. Express 15, 10091-10096 (2007).

29. C. Doerr, L. Chen, M. Rasras, Y. Chen, J. Weiner, and M. Earnshaw, "Diplexer with integrated filters and photodetector in Ge-Si using - X and M directions in a grating coupler", IEEE Photonic. Tech. L. 21, 1698-1700 (2009).

30. L. Xu, Q. Li, N. Ophir, K. Padmaraju, L. Luo, L. Chen, M. Lipson, and K. Bergman, "Colorless optical network unit based on silicon photonic components for WDM PON", IEEE Photonic. Tech. L. 24, 1372-1374 (2012).

31. J. Xiao, X. Liu, and X. Sun, "Design of an ultracompact MMI wavelength demultiplexer in slot waveguide structures", Opt. Express 15, 8300-8308 (2007).

32. H. Yi, Y. Huang, X. Wang, and Z. Zhou, "Ultra-short silicon MMI duplexer", Proceedings of SPIE - The International Society for Optical Engineering 8564, 10-16 (2012).

33. Y. Ma, P. Magill, T. Baehr-Jones, and M. Hochberg, "Design and optimization of a novel silicon-on-insulator wavelength diplexer", Opt. Express 22, 21521-21528 (2014).

34. A. Piggott, J. Lu, K. Lagoudakis, J. Petykiewicz, T. Babinec, and J. Vučković, "Inverse design and demonstration of a compact and broadband on-chip wavelength demultiplexer", Nat. Photonics 9, 374-377 (2015).

35. Y. Shi, S. Anand, and S. He, "Design of a polarization insensitive triplexer using directional couplers based on submicron silicon rib waveguides", J. Lightwave Technol. 27, 1443-1447 (2009).

36. T. Holmgaard, Z. Chen, S. Bozhevolnyi, L. Markey, and A. Dereux, "Design and characterization of dielectric-loaded plasmonic directional couplers", J. Lightwave Technol. 27, 5521-5528 (2009).

37. M. Koshiba, "Wavelength division multiplexing and demultiplexing with photonic crystal waveguide couplers", J. Lightwave Technol. 19, 19701975 (2001).

38. A. Kumar and S. Aditya, "Performance of S-bends for integrated-optic waveguides", Microw. Opt. Techn. Let. 19, 289-292 (1998). 